\title{
Contraction mapping principle in partially ordered quasi metric space concerning to $w$-distances
}

\author{
Rahma Zuhra ${ }^{\mathrm{a}, \mathrm{b}, *}$, Mohd Salmi Md Noorania ${ }^{\mathrm{a}}$ Fawzia Shaddad ${ }^{\mathrm{c}}$ \\ a School of Mathematical Sciences, Faculty of Science and Technology University Kebangsaan Malaysia, UKM, 43600 Bangi, Malaysia. \\ ${ }^{b}$ Department of Mathematics, Faculty of Mathematics and Natural Sciences, Syiah Kuala University, 23111 Banda Aceh, Indonesia. \\ ${ }^{c}$ Department of Mathematics, Sana'a University, Yemen.
}

Communicated by W. Shatanawi

\begin{abstract}
The fixed point theorems in various contraction mappings have been provided by many researchers. Some of them used certain functions in mapping to guarantee the existence of fixed point. The purpose of this paper is to present some fixed point result on contraction mapping in partially ordered quasi-metric space that applying a $w$-distance. The generalized altering distance function on the mapping plays a role in theorems. The results extend some well-known results in the references. We also improve these new results to the common fixed point. (C)2017 All rights reserved.
\end{abstract}

Keywords: Fixed point, $w$-distance, a generalized altering distance function, common fixed point. 2010 MSC: 47H10, 54H25.

\section{Introduction and preliminaries}

The availability of references about a generalization of the Banach contraction principle has been studied in many branches of research. For instance the weak contraction mapping has been researched by many authors in different spaces, see $[1,3,4,21,23,27]$. In $[2,6,7,10,15,18,19,22,29]$, they have proved some results of fixed point theorems on weak contractions mappings in metric spaces. Among the references, they have applied an altering distance function. Khan et al. [15] introduced this concept to handle functions which changes the distance between two points in metric space. Recently, Su [28] has expanded the concept and introduced a generalized altering distance function as follows.

Definition 1.1 ([28]). A generalized altering distance function is a function $\psi:[0, \infty) \rightarrow[0, \infty)$ which satisfies:

(a) $\psi$ is nondecreasing;

(b) $\psi(t)=0$ if and only if $t=0$.

\footnotetext{
*Corresponding author

Email addresses: r4hm4@siswa.ukm.edu.my (Rahma Zuhra), msn@ukm.edu.my (Mohd Salmi Md Noorani), fzsh99@gmail.com (Fawzia Shaddad)
}

doi:10.22436/jnsa.010.02.31

Received 2016-10-24 
In 2010, Harjani and Sadarangani [10] have proved some fixed point theorems for weak contraction and generalized contractions in partially ordered metric spaces by using the altering distance function. Theorems in $[6,18]$ were updated by these results.

Theorem 1.2 ([10]). Let $(X, \preceq)$ be a partially ordered set and suppose that there exists a metric $\mathrm{d} \in \mathrm{X}$ such that $(\mathrm{X}, \mathrm{d})$ is a complete metric space. Let $\mathrm{f}: \mathrm{X} \rightarrow \mathrm{X}$ be a continuous and nondecreasing mapping such that

$$
\psi(d(f(x), f(y)) \leqslant \psi(d(x, y))-\phi(d(x, y)) \text { for } x \geqslant y,
$$

where $\psi$ and $\phi$ are altering distance functions. If there exists $x_{0} \in X$ with $x_{0} \preceq f\left(x_{0}\right)$, then $f$ has a fixed point.

$\mathrm{Su}$ [28] established a new fixed point theorem by using the generalized altering distance function. Then, Shaddad et al.[22] has made general results not only for fixed point but also for coupled coincidence point in partially ordered metric space. The important results of them are in the following.

Theorem 1.3 ([22]). Let $(X, d, \preceq)$ be a complete partially ordered metric space. Let $f: X \rightarrow X$ be a mapping which obeys the following conditions:

(1) there exist a generalized altering distance function $\psi$, an upper semi-continuous function $\varphi:[0, \infty) \rightarrow[0, \infty)$ and a lower semi-continuous function $\phi:[0, \infty) \rightarrow[0, \infty)$ such that

$$
\psi(d(f(x), f(y))) \leqslant \varphi(d(x, y))-\phi(d(x, y)) \text { for } x \asymp y,
$$

where $\varphi(0)=\phi(0)=0$ and $\psi(t)-\varphi(t)+\phi(t)>0$ for all $t>0$;

(2) there exists $x_{0} \in X$ such that $x_{0} \asymp f x_{0}$;

(3) $f$ is nondecreasing;

(4) (a) either $f$ is continuous or

(b) if $x_{n} \rightarrow x$ when $n \rightarrow \infty$ in $x$, then $x_{n} \asymp x$ for all $n$.

Then $\mathrm{f}$ has a fixed point. Moreover, if for each $\mathrm{x}, \mathrm{y} \in \mathrm{X}$ there exists $z \in \mathrm{X}$ which is comparable to $x$ and $y$ then the fixed point is unique.

The concept of $w$-distance was initiated by Kada et al. [14]. Several researchers involve this notion to obtain some fixed point results not only in usual metric spaces but also in partially ordered metric spaces. Now, we recall the definition of $w$-distance.

Definition $1.4([14])$. A $w$-distance on a metric space $(X, d)$ is a function $q: X \times X \rightarrow[0, \infty)$ satisfying the following conditions:

(w1) $q(x, y) \leqslant q(x, z)+q(z, y)$ for all $x, y, z \in X$;

(w2) $\mathrm{q}(x,):. X \rightarrow[0, \infty)$ is a lower-semicontinuous for all $x \in X$ (i.e., if $x \in X$ and $y_{n} \rightarrow y$, then $\left.q(x, y) \leqslant \lim _{n \rightarrow \infty} \inf _{n} q\left(x, y_{n}\right)\right)$

(w3) for each $\epsilon>0$ there exists $\delta>0$ such that $q(x, y) \leqslant \delta$ and $q(x, z) \leqslant \delta$ imply $d(y, z) \leqslant \epsilon$ for all $x, y, z \in X$.

Note that in general for $x, y \in X, q(x, y) \neq q(y, x)$ and not either the implications $q(x, y)=0 \Leftrightarrow x=y$ is necessarily true [16].

Meanwhile, $\Omega$-distance as a generalized form of $w$-distance was also studied. This notion has introduced by Saadati et al. [21] in 2010. They have proved fixed point theorems on a complete partially ordered G-metric space as well. Then, $\Omega$-distance function was expanded by Gholizadeh et al. [8] and they gave an application in integral equations. In 2013, Shatanawi and Pitea [26] have used the concept of $\Omega$-distance to establish some common coupled fixed point results. They continued their work to construct and prove some fixed and coupled fixed point theorems for a nonlinear contraction [25] . Other interesting results are gained by Shatanawi et al. [23, 24] in 2016. Shatanawi et al. [24] have introduced new fixed point and common fixed point for mappings of the cyclic form of $\Omega$-distance in G-metric space. Next, 
Shatanawi et al. [23] improved the usability of this notion by introducing a new contraction mapping called $\Omega$-suzuki-construction in g-metric space.

Now, the following definitions and lemmas which have been stated in metric space $[3,12,22]$ will be used in quasi metric space. Following the terminology of [3], by a quasi metric on a nonempty set $X$ we mean a function $d: X \times X \rightarrow[0, \infty)$ such that

(i) $d(x, y)=d(y, x)$ if and only if $x=y$ for all $x, y \in X$ and

(ii) $d(x, y) \leqslant d(x, z)+d(z, y)$ for all $x, y, z \in X$.

A quasi metric space is a pair $(X, d)$ such that $X$ is a set and $d$ is a quasi metric on $X$. Quasi metric satisfies the triangle inequality but is not symmetric. It can be regarded as an "asymmetric metric". In fact, quasi metric space is more comprehensive than metric space. Given a quasi metric $d$ on $X$, the function $d^{\prime}$ defined by $d^{\prime}(x, y)=d(y, x)$ for all $x, y \in X$, is also a quasi metric on $X$. The function $d^{s}$ defined by $d^{s}(x, y)=\max \{d(x, y), d(y, x)\}$ for all $x, y \in X$ is a metric on $X$. A quasi metric space $(X, d)$ is called complete if every Cauchy sequence $\left\{x_{n}\right\}_{n \in P}$ in the metric space $\left(X, d^{s}\right)$ is convergent [3].

Several results from Kada et al. [14] were continued by Park [17] to quasi-metric spaces. Next, Latif and Al-Mezel [16] also have proved some fixed point theorems in complete quasi-metric spaces. The purpose of this paper is to prove some fixed point theorems in partially ordered quasi-metric spaces by using a $w$-distance. We discuss the existence of fixed point by extending the results of Harjani and Sadarangani [10], Imdad and Rouzkard [12], Shaddad et al. [22], Su [28], and Rouzkard et al. [20]. Then, we apply the results of Alegre et al. [3] to prove the main theorem. After that, we also provide an example that satisfies our theorems. Furthermore, we improve new result to common fixed points that expands the results from Ciric et al. [4] and Ilic and Rakocevic [11]. However, in this paper, we prove the shorter way to common fixed point, that is motivated by the fact that a fixed point of any map on metric spaces can be viewed as a common fixed point of that mapping.

\section{Main results}

Throughout this paper, we denote by $\mathbb{R}$ and $\mathbb{N}$ the sets of real numbers and non-negative integers, respectively. The definitions and lemmas are crucial in the proof of our main results. We apply the notions in a metric space for a quasi-metric space in the following.

Definition 2.1 ([12]). Let $X$ be a nonempty set. Then $(X, d, \preceq)$ is called a partially ordered quasi metric space if

(i) $(X, \preceq)$ is a partially ordered set; and

(ii) $(X, d)$ is a quasi metric space.

Definition $2.2([12])$. Let $(X, \preceq)$ be a partially ordered set. Then

(a) elements $x, y \in X$ are called comparable with respect to " $\preceq$ " if either $x \preceq y$ or $y \preceq x$ and we write $x \asymp y$;

(b) a mapping $f: X \rightarrow X$ is called nondecreasing with respect to " $\preceq$ " if $x \preceq y$ implies $f(x) \preceq f(y)$.

Lemma 2.3 ([3]). Let $q$ be an $w$-distance on a quasi metric space $(X, d)$ and $\left\{x_{n}\right\}$ be a sequence in $X$ such that for each $\epsilon>0$ there exists $n_{0} \in \mathbb{N}$ whenever $m>n>n_{0}$ implies $q\left(x_{n}, x_{m}\right) \leqslant \epsilon\left(\right.$ or $\left.\lim _{m, n \rightarrow \infty} q\left(x_{n}, x_{m}\right)=0\right)$. Then $\left\{x_{n}\right\}$ is a Cauchy sequence.

Lemma 2.4 ([3]). If $q$ is an $w$-distance on a quasi metric space $(X, d)$, then for each $\epsilon>0$ there exists $\delta>0$ such that $\mathrm{q}(\mathrm{x}, \mathrm{y}) \leqslant \delta$ and $\mathrm{q}(\mathrm{x}, z) \leqslant \delta$ imply $\mathrm{d}^{\mathrm{s}}(\mathrm{y}, z) \leqslant \epsilon$.

Now, we state the main result as follows. 
Theorem 2.5. Let $(\mathrm{X}, \mathrm{d}, \preceq)$ be a complete partially ordered quasi metric space equipped with $\boldsymbol{w}$-distance $\mathrm{q}$ and $f: X \rightarrow X$ has the following conditions:

(1) there exists $x_{0} \in X$ such that $x_{0} \asymp \mathrm{fx}_{0}$;

(2) there exist a generalized altering distance function $\psi$, an upper semi-continuous function $\varphi:[0, \infty) \rightarrow[0, \infty)$ and a lower semi-continuous function $\phi:[0, \infty) \rightarrow[0, \infty)$ such that

$$
\psi(q(f x, f y)) \leqslant \varphi\left(M_{x, y}\right)-\phi\left(M_{x, y}\right) \text { for } x \asymp y,
$$

where $M_{x, y}=\max \left\{q(x, y), q(x, f x), q(y, f y), \frac{1}{2}[q(x, f y)-q(y, f x)]\right\}, \varphi(0)=\phi(0)=0$, and $\psi(t)-\varphi(t)+$ $\phi(\mathrm{t})>0$ for all $\mathrm{t}>0$;

(3) $f$ is nondecreasing and continuous.

Then $\mathrm{f}$ has a fixed point.

Proof. By condition (1), there exists a point $x_{0}$ such that $x_{0} \asymp f x_{0}$. Take $x_{1} \in X$ such that $x_{1}=f x_{0}$, that is, $x_{0} \asymp x_{1}$. Now, take $x_{2}=f x_{1}$. In general, we can define $f x_{n}=x_{n+1}$ for all $n \in \mathbb{N}$. Using condition (3), i.e., $f$ is a nondecreasing, then we obtain that $f x_{0} \asymp f x_{1}$ or $x_{1} \asymp x_{2}$. Continuing this process inductively, we have $x_{n} \asymp x_{n+1}$ for all $n \in \mathbb{N}$, i.e.,

$$
x_{0} \asymp x_{1} \asymp x_{2} \asymp \cdots \asymp x_{n} \asymp \cdots
$$

That is,

$$
x_{0} \preceq x_{1} \preceq x_{2} \preceq \cdots \preceq x_{n} \preceq \cdots
$$

or

$$
x_{0} \succeq x_{1} \succeq x_{2} \succeq \cdots \succeq x_{n} \succeq \cdots
$$

It means, $x_{n} \asymp x_{n+1}$ can be written as $x_{n} \asymp x_{m}$ for each $n, m \in \mathbb{N}$.

Now, if $x_{n}=x_{n+1}$ for some $n \in \mathbb{N}$, then $f$ has a fixed point. Assume that $x_{n} \neq x_{n+1}$ for all $n \in \mathbb{N}$. Now, we proceed to show that $\left\{\mathbf{q}\left(x_{n}, x_{n+1}\right)\right\}$ is a decreasing sequence. In contrary, suppose $\left\{\mathbf{q}\left(x_{n}, x_{n+1}\right)\right\}$ is a nondecreasing sequence. From condition (2), we have

$$
\psi\left(q\left(x_{n+1}, x_{n+2}\right)\right)=\psi\left(q\left(f x_{n}, f x_{n+1}\right)\right) \leqslant \varphi\left(M_{x_{n}, x_{n+1}}\right)-\phi\left(M_{x_{n}, x_{n+1}}\right),
$$

where

$$
\begin{aligned}
M_{x_{n}, x_{n+1}} & =\max \left\{q\left(x_{n}, x_{n+1}\right), q\left(x_{n}, f x_{n}\right), q\left(x_{n+1}, f x_{n+1}\right), \frac{1}{2}\left[q\left(x_{n}, f x_{n+1}\right)-q\left(x_{n+1}, f x_{n}\right)\right]\right\} \\
& =\max \left\{q\left(x_{n}, x_{n+1}\right), q\left(x_{n}, x_{n+1}\right), q\left(x_{n+1}, x_{n+2}\right), \frac{1}{2}\left[q\left(x_{n}, x_{n+2}\right)-q\left(x_{n+1}, x_{n+1}\right)\right]\right\} \\
& =\max \left\{q\left(x_{n}, x_{n+1}\right), q\left(x_{n+1}, x_{n+2}\right), \frac{1}{2}\left[q\left(x_{n}, x_{n+2}\right)-q\left(x_{n+1}, x_{n+1}\right)\right]\right\} .
\end{aligned}
$$

We face three cases.

Case 1. $M_{x_{n}, x_{n+1}}=q\left(x_{n}, x_{n+1}\right)$, then from (2.1) we obtain

$$
\psi\left(q\left(x_{n+1}, x_{n+2}\right)\right) \leqslant \varphi\left(q\left(x_{n}, x_{n+1}\right)\right)-\phi\left(q\left(x_{n}, x_{n+1}\right)\right)<\psi\left(q\left(x_{n}, x_{n+1}\right)\right) .
$$

Since $\psi$ is nondecreasing, so $q\left(x_{n+1}, x_{n+2}\right)<q\left(x_{n}, x_{n+1}\right)$ is a contradiction.

Case 2. $M_{x_{n}, x_{n+1}}=q\left(x_{n+1}, x_{n+2}\right)$, then from (2.1) we obtain

$$
\psi\left(q\left(x_{n+1}, x_{n+2}\right)\right) \leqslant \varphi\left(q\left(x_{n+1}, x_{n+2}\right)\right)-\phi\left(q\left(x_{n+1}, x_{n+2}\right)\right)<\psi\left(q\left(x_{n+1}, x_{n+2}\right)\right) .
$$

Since $\psi$ is nondecreasing, so $q\left(x_{n+1}, x_{n+2}\right)<q\left(x_{n+1}, x_{n+2}\right)$ is a contradiction. 
Case 3. $M_{x_{n}, x_{n+1}}=\frac{1}{2}\left[q\left(x_{n}, x_{n+2}\right)-q\left(x_{n+1}, x_{n+1}\right)\right]$, then from (2.1) we obtain

$$
\begin{aligned}
\psi\left(q\left(x_{n+1}, x_{n+2}\right)\right) & \leqslant \varphi\left(\frac{1}{2}\left[q\left(x_{n}, x_{n+2}\right)-q\left(x_{n+1}, x_{n+1}\right)\right]\right)-\phi\left(\frac{1}{2}\left[q\left(x_{n}, x_{n+2}\right)-q\left(x_{n+1}, x_{n+1}\right)\right]\right) \\
& <\psi\left(\frac{1}{2}\left[q\left(x_{n}, x_{n+2}\right)-q\left(x_{n+1}, x_{n+1}\right)\right]\right) .
\end{aligned}
$$

Since $\psi$ is nondecreasing, so

$$
\begin{aligned}
q\left(x_{n+1}, x_{n+2}\right) & <\frac{1}{2}\left[q\left(x_{n}, x_{n+2}\right)-q\left(x_{n+1}, x_{n+1}\right)\right], \\
2 q\left(x_{n+1}, x_{n+2}\right) & <q\left(x_{n}, x_{n+2}\right)-q\left(x_{n+1}, x_{n+1}\right)<q\left(x_{n}, x_{n+2}\right) \leqslant q\left(x_{n}, x_{n+1}\right)+q\left(x_{n+1}, x_{n+2}\right), \\
q\left(x_{n+1}, x_{n+2}\right) & \leqslant q\left(x_{n}, x_{n+1}\right)
\end{aligned}
$$

is also a contradiction.

As a result of cases 1,2 , and 3 , we conclude that $\left\{q\left(x_{n}, x_{n+1}\right)\right\}$ is a decreasing sequence. In particular, there exists $r \geqslant 0$ such that $\lim _{n \rightarrow \infty} q\left(x_{n}, x_{n+1}\right)=r$. Assume that $r \neq 0$. By using the properties of $\psi, \varphi$, and $\phi$ and taking $n \rightarrow \infty$ we obtain

- if $M_{x_{n}, x_{n+1}}=q\left(x_{n}, x_{n+1}\right)$ or $q\left(x_{n+1}, x_{n+2}\right)$, then from (2.2) and (2.3), we gain $\psi(r) \leqslant \varphi(r)-\phi(r)$. Therefore $\psi(r)-\varphi(r)+\phi(r) \leqslant 0$ is a contradiction with condition (2). Then $r=0$ and $\lim _{n \rightarrow \infty} q\left(x_{n}, x_{n+1}\right)=0$.

- if $M_{x_{n}, x_{n+1}}=\frac{1}{2}\left[q\left(x_{n}, x_{n+2}\right)-q\left(x_{n+1}, x_{n+1}\right)\right]$, then from (2.4), we have

$$
\begin{aligned}
\psi\left(q\left(x_{n+1}, x_{n+2}\right)\right) & \leqslant \varphi\left(\frac{1}{2}\left[q\left(x_{n}, x_{n+2}\right)-q\left(x_{n+1}, x_{n+1}\right)\right]\right)-\phi\left(\frac{1}{2}\left[q\left(x_{n}, x_{n+2}\right)-q\left(x_{n+1}, x_{n+1}\right)\right]\right) \\
& <\psi\left(\frac{1}{2}\left[q\left(x_{n}, x_{n+2}\right)-q\left(x_{n+1}, x_{n+1}\right)\right]\right) .
\end{aligned}
$$

From (2.5), we get

$$
\begin{aligned}
\psi\left(q\left(x_{n+1}, x_{n+2}\right)\right) & <\psi\left(\frac{1}{2}\left[q\left(x_{n}, x_{n+2}\right)-q\left(x_{n+1}, x_{n+1}\right)\right]\right) \\
& \leqslant \psi\left(\frac{1}{2}\left[q\left(x_{n}, x_{n+1}\right)+q\left(x_{n+1}, x_{n+1}\right)+q\left(x_{n+1}, x_{n+2}\right)-q\left(x_{n+1}, x_{n+1}\right)\right]\right) \\
& =\psi\left(\frac{1}{2}\left[q\left(x_{n}, x_{n+1}\right)+q\left(x_{n+1}, x_{n+2}\right)\right]\right) .
\end{aligned}
$$

From (2.5), (2.6), and taking $n \rightarrow \infty$, we obtain

$$
\begin{aligned}
& \psi(r) \leqslant \psi\left(\left(\frac{1}{2}\left[\lim _{n \rightarrow \infty}\left(q\left(x_{n}, x_{n+2}\right)-q\left(x_{n+1}, x_{n+1}\right)\right)\right]\right)\right) \leqslant \psi\left(\frac{1}{2}(r+r)\right) \\
& \psi(r) \leqslant \psi\left(\left(\frac{1}{2}\left[\lim _{n \rightarrow \infty}\left(q\left(x_{n}, x_{n+2}\right)-q\left(x_{n+1}, x_{n+1}\right)\right)\right]\right)\right) \leqslant \psi\left(\frac{1}{2}(2 r)\right) \\
& \psi(r) \leqslant \psi\left(\left(\frac{1}{2}\left[\lim _{n \rightarrow \infty}\left(q\left(x_{n}, x_{n+2}\right)-q\left(x_{n+1}, x_{n+1}\right)\right)\right]\right)\right) \leqslant \psi(r) .
\end{aligned}
$$

Since $\psi$ is nondecreasing, we obtain

$$
\left.\frac{1}{2}\left[\lim _{n \rightarrow \infty}\left(q\left(x_{n}, x_{n+2}\right)-q\left(x_{n+1}, x_{n+1}\right)\right)\right]=r, \quad \lim _{n \rightarrow \infty}\left(q\left(x_{n}, x_{n+2}\right)-q\left(x_{n+1}, x_{n+1}\right)\right)\right]=2 r .
$$

We go back to (2.5), then we gain

$$
\psi(r) \leqslant \varphi\left(\frac{1}{2}(2 r)\right)-\phi\left(\frac{1}{2}(2 r)\right), \text { or } \psi(r) \leqslant \varphi(r)-\phi(r) .
$$

Therefore $\psi(r)-\varphi(r)+\phi(r) \leqslant 0$ is a contradiction with condition (2) in our theorem. Then $r=0$ and $\lim _{n \rightarrow \infty} q\left(x_{n}, x_{n+1}\right)=0$. 
Next, we show that $\left\{x_{n}\right\}$ is Cauchy sequence. That is, if we choose an arbitrary $\epsilon>0$ and let $\delta \in(0, \epsilon)$ then there is $n_{0} \in \mathbb{N}$ such that $q\left(x_{n}, x_{m}\right)<\delta$, whenever $m>n \geqslant n_{0}$. In contrary,

$$
q\left(x_{n}, x_{m}\right) \geqslant \delta \text { whenever } m>n \geqslant n_{0} .
$$

Furthermore, corresponding to $n$, we can choose $m$ is the smallest integer with $m>n \geqslant n_{0}$ and satisfying (2.7). Then

$$
q\left(x_{n}, x_{m-1}\right)<\delta \text { for all } m>n \text {. }
$$

Since $x_{n} \asymp x_{m}$ for all $m>n$, then from condition (2) in our theorem we get

$$
\psi\left(q\left(x_{n}, x_{m}\right)\right)=\psi\left(q\left(f x_{n-1}, f x_{m-1}\right)\right) \leqslant \varphi\left(M_{x_{n-1}, x_{m-1}}\right)-\phi\left(M_{x_{n-1}, x_{m-1}}\right),
$$

where

$$
\begin{aligned}
M_{x_{n-1}, x_{m-1}} & =\max \left\{q\left(x_{n-1}, x_{m-1}\right), q\left(x_{n-1}, f x_{n-1}\right), q\left(x_{m-1}, f x_{m-1}\right), \frac{1}{2}\left[q\left(x_{n-1}, f x_{m-1}\right)-q\left(x_{m-1}, f x_{n-1}\right)\right]\right\} \\
& =\max \left\{q\left(x_{n-1}, x_{m-1}\right), q\left(x_{n-1}, x_{n}\right), q\left(x_{m}-1, x_{m}\right), \frac{1}{2}\left[q\left(x_{n-1}, x_{m}\right)-q\left(x_{m-1}, x_{n}\right)\right]\right\} .
\end{aligned}
$$

Now, we have two cases.

- If $M_{x_{n-1}, x_{m-1}}=q\left(x_{n-1}, x_{m-1}\right)$, then

$$
\psi\left(q\left(x_{n}, x_{m}\right)\right) \leqslant \varphi\left(q\left(x_{n-1}, x_{m-1}\right)\right)-\phi\left(q\left(x_{n-1}, x_{m-1}\right)\right)<\psi\left(q\left(x_{n-1}, x_{m-1}\right)\right) .
$$

Since $\psi$ is nondecreasing, then

$$
q\left(x_{n}, x_{m}\right)<q\left(x_{n-1}, x_{m-1}\right) \leqslant q\left(x_{n-1}, x_{n}\right)+q\left(x_{n}, x_{m-1}\right) .
$$

From (2.7), (2.9), and by taking $n \rightarrow \infty$, we obtain

$$
\delta \leqslant \lim _{n \rightarrow \infty} q\left(x_{n}, x_{m}\right)<\lim _{n \rightarrow \infty} q\left(x_{n-1}, x_{m-1}\right) \leqslant \lim _{n \rightarrow \infty} q\left(x_{n-1}, x_{n}\right)+\lim _{n \rightarrow \infty} q\left(x_{n}, x_{m-1}\right)<\delta .
$$

Thus,

$$
\lim _{n \rightarrow \infty} q\left(x_{n}, x_{m}\right)=\delta
$$

Moreover, we rewrite

$$
q\left(x_{n-1}, x_{m-1}\right) \leqslant q\left(x_{n-1}, x_{n}\right)+q\left(x_{n}, x_{m-1}\right) .
$$

From (2.9) and (2.11) and by taking $n \rightarrow \infty$, we gain

$$
\delta \leqslant \lim _{n \rightarrow \infty} q\left(x_{n-1}, x_{m-1}\right) \leqslant \lim _{n \rightarrow \infty} q\left(x_{n-1}, x_{n}\right)+\lim _{n \rightarrow \infty} q\left(x_{n}, x_{m-1}\right)=0+\delta .
$$

Thus

$$
\lim _{n \rightarrow \infty} q\left(x_{n-1}, x_{m-1}\right)=\delta .
$$

- If $M_{x_{n-1}, x_{m-1}}=\frac{1}{2}\left[q\left(x_{n-1}, x_{m}\right)-q\left(x_{m-1}, x_{n}\right)\right]$, then

$$
\begin{aligned}
\psi\left(q\left(x_{n}, x_{m}\right)\right) & \leqslant \varphi\left(\frac{1}{2}\left[q\left(x_{n-1}, x_{m}\right)-q\left(x_{m-1}, x_{n}\right)\right]\right)-\phi\left(\frac{1}{2}\left[q\left(x_{n-1}, x_{m}\right)-q\left(x_{m-1}, x_{n}\right)\right]\right) \\
& <\psi\left(\frac{1}{2}\left[q\left(x_{n-1}, x_{m}\right)-q\left(x_{m-1}, x_{n}\right)\right]\right) .
\end{aligned}
$$

Since $\psi$ is nondecreasing, then

$$
q\left(x_{n}, x_{m}\right)<\frac{1}{2}\left[q\left(x_{n-1}, x_{m}\right)-q\left(x_{m-1}, x_{n}\right)\right]<\frac{1}{2} q\left(x_{n-1}, x_{m}\right) .
$$


Thus

$$
2 q\left(x_{n}, x_{m}\right)<q\left(x_{n-1}, x_{m}\right) \leqslant q\left(x_{n-1}, x_{n}\right)+q\left(x_{n}, x_{m-1}\right)+q\left(x_{m-1}, x_{m}\right) .
$$

Let $n \rightarrow \infty$ in (2.14), then

$$
\begin{aligned}
2 \lim _{n \rightarrow \infty} q\left(x_{n}, x_{m}\right)<\lim _{n \rightarrow \infty} q\left(x_{n-1}, x_{m}\right) & \leqslant \lim _{n \rightarrow \infty} q\left(x_{n-1}, x_{n}\right)+\lim _{n \rightarrow \infty} q\left(x_{n}, x_{m-1}\right)+\lim _{n \rightarrow \infty} q\left(x_{m-1}, x_{m}\right), \\
\delta & \leqslant 2 \delta<\lim _{n \rightarrow \infty} q\left(x_{n-1}, x_{m}\right) \leqslant \delta, \\
\delta & \leqslant \lim _{n \rightarrow \infty} q\left(x_{n-1}, x_{m}\right) \leqslant \delta .
\end{aligned}
$$

So

$$
\lim _{n \rightarrow \infty} q\left(x_{n-1}, x_{m}\right)=\delta
$$

From (2.13), we have

$$
2 q\left(x_{n}, x_{m}\right)<q\left(x_{n-1}, x_{m}\right)-q\left(x_{m-1}, x_{n}\right)<q\left(x_{n-1}, x_{m}\right) .
$$

Combining (2.10) and (2.15) and taking $n \rightarrow \infty$, then

$$
\begin{aligned}
2 \delta & \leqslant \delta-\lim _{n \rightarrow \infty} q\left(x_{m-1}, x_{n}\right) \leqslant \delta, \\
\delta & \leqslant \delta-\lim _{n \rightarrow \infty} q\left(x_{m-1}, x_{n}\right) \leqslant \delta, \\
0 & \leqslant \lim _{n \rightarrow \infty} q\left(x_{m-1}, x_{n}\right) \leqslant 0 .
\end{aligned}
$$

Thus

$$
\lim _{n \rightarrow \infty} q\left(x_{m-1}, x_{n}\right)=0 .
$$

So, by taking into account (2.15), (2.16), and $n \rightarrow \infty$, we deduce

$$
\lim _{n \rightarrow \infty}\left(\frac{1}{2}\left[q\left(x_{n-1}, x_{m}\right)-q\left(x_{m-1}, x_{n}\right)\right]\right)=\frac{1}{2}(\delta-0)=\frac{1}{2} \delta .
$$

Finally from (2.8), (2.10), (2.12), (2.17), and taking $n \rightarrow \infty$, we conclude $\psi(\delta) \leqslant \varphi(M)-\phi(M)$, where $M=\max \left\{\delta, 0, \frac{1}{2} \delta\right\}=\delta$.

So, $\psi(\delta) \leqslant \varphi(\delta)-\phi(\delta)$ or $\psi(\delta)-\varphi(\delta)+\phi(\delta) \leqslant 0$. This is contradiction since $\delta>0$. Hence

$$
\mathrm{q}\left(\mathrm{x}_{\mathrm{n}}, \mathrm{x}_{\mathrm{m}}\right)<\delta \text { for } \mathrm{m}>\mathrm{n} \geqslant \mathrm{n}_{0}, \mathrm{n}_{0} \in \mathbb{N} .
$$

In particular, we have $q\left(x_{n_{0}}, x_{n}\right)<\delta$ and $q\left(x_{n_{0}}, x_{m}\right)<\delta$, whenever $m>n \geqslant n_{0}$. Thus from Lemma 2.4, we get $d^{s}\left(x_{n}, x_{m}\right) \leqslant \epsilon$. Hence $\left\{x_{n}\right\}$ is Cauchy sequence in $\left(X, d^{s}\right)$. Therefore $\left\{x_{n}\right\}$ is also Cauchy sequence in $(X, d)$. Since $(X, d)$ is complete then $\left\{x_{n}\right\}$ converges, i.e., there exists $x^{*} \in X$ such that $d\left(x_{n}, x^{*}\right) \rightarrow 0$.

Now we want to show that $q\left(x_{n}, x^{*}\right) \rightarrow 0$. Following (2.18), we can choose an arbitrary $\epsilon>0$ such that $\mathrm{q}\left(\mathrm{x}_{\mathrm{n}}, \mathrm{x}_{\mathrm{m}}\right)<\epsilon$ for $\mathrm{m}>\mathrm{n}>\mathrm{n}_{0}, \mathrm{n}_{0} \in \mathbb{N}$. Let us fix $\mathrm{n}>\mathrm{n}_{0}$, from Definition 1.4 and for $n$ sufficiently large

$$
q\left(x_{n}, x^{*}\right) \leqslant \liminf _{m \rightarrow \infty} q\left(x_{n}, x_{m}\right) \leqslant q\left(x_{n}, x_{m}\right)+\epsilon<2 \epsilon .
$$

Since $\epsilon>0$ is arbitrary, then

$$
\mathrm{q}\left(x_{n}, x^{*}\right) \rightarrow 0
$$

Now, we show that $f x^{*}=x^{*}$.

We have $q\left(x_{n}, x^{*}\right) \rightarrow 0$ from (2.19), then $x_{n} \rightarrow x^{*}$ as $n \rightarrow \infty$. By using condition (3) in our theorem, $f$ is continuous. That is, $f x_{n} \rightarrow f x^{*}$ as $n \rightarrow \infty$, then $x_{n} \rightarrow f x^{*}$ as $n \rightarrow \infty$. So, $q\left(x_{n}, f x^{*}\right) \rightarrow 0$. From Lemma 2.4, for all $\epsilon>0$, there exists $\delta \in(0, \epsilon)>0$ such that $\mathrm{q}\left(x_{n}, x^{*}\right)<\delta, q\left(x_{n}, f x^{*}\right)<\delta$ imply $d^{s}\left(x^{*}, f x^{*}\right) \leqslant \epsilon$. Then $d^{s}\left(x^{*}, f x^{*}\right)=0$, so that $f x^{*}=x^{*}$. Hence $x^{*}$ is a fixed point of $f$. 
Remark 2.6. We note that one of $\mathrm{M}_{x, y}$ as follows

$$
\begin{aligned}
\frac{1}{2}[q(x, f y)-q(y, f x)] \leqslant \frac{1}{2}[q(x, f y)] \leqslant \frac{(q(x, y)+q(y, f y))}{2} & \leqslant 2\left(\frac{\max [q(x, y), q(y, f y)]}{2}\right) \\
& =\max \{q(x, y), q(y, f y)\} .
\end{aligned}
$$

Thus, $\bar{M}_{x, y}=\max \{q(x, y), q(x, f x), q(y, f y)\}$. Furthermore, we obtain the following corollary.

Corollary 2.7. Let $(\mathrm{X}, \mathrm{d}, \preceq)$ be a complete partially ordered quasi metric space equipped with $w$-distance $\mathrm{q}$. Let $\mathrm{f}: \mathrm{X} \rightarrow \mathrm{X}$ which has the following conditions:

(1) there exists $x_{0} \in X$ such that $x_{0} \asymp f x_{0}$;

(2) there exist a generalized altering distance function $\psi$, an upper semi-continuous function $\varphi:[0, \infty) \rightarrow[0, \infty)$ and a lower semi-continuous function $\phi:[0, \infty) \rightarrow[0, \infty)$ such that

$$
\psi(q(f x, f y)) \leqslant \varphi\left(\bar{M}_{x, y}\right)-\phi\left(\bar{M}_{x, y}\right) \text { for } x \asymp y,
$$

where $\bar{M}_{x, y}=\max \{q(x, y), q(x, f x), q(y, f y)\}, \varphi(0)=\phi(0)=0$ and $\psi(t)-\varphi(t)+\phi(t)>0$ for all $t>0$;

(3) $f$ is nondecreasing and continuous.

Then $\mathrm{f}$ has a fixed point.

If we set $\varphi=\psi$ and $\phi$ is a generalized altering distance function in Theorem 2.5, we obtain generalization of the results of Imdad and Rouzkard [12] in the following corollary.

Corollary 2.8. Let $(\mathrm{X}, \mathrm{d}, \preceq)$ be a complete partially ordered quasi metric space equipped with $\mathrm{w}$-distance $\mathrm{q}$. Let $\mathrm{f}$ be a self-mapping on $\mathrm{X}$ which has the following conditions:

(1) there exists $x_{0} \in X$ such that $x_{0} \asymp f x_{0}$;

(2) there exist two generalized altering distance functions $\psi, \phi$, such that

$$
\psi(q(f x, f y)) \leqslant \psi\left(M_{x, y}\right)-\phi\left(M_{x, y}\right), \quad \text { for all } x \asymp y,
$$

where $M_{x, y}=\max \left\{q(x, y), q(x, f x), q(y, f y), \frac{1}{2}[q(x, f y)-q(y, f x)]\right\}, \phi(0)=0$ and $\phi(t)>0, \quad$ for all $t>$ 0 ;

(3) $f$ is nondecreasing and continuous.

Then $\mathrm{f}$ has a fixed point.

Remark 2.9. We do not use the condition (4)-(b) and uniqueness from Theorem 1.3 to Theorem 2.5 because there are two points of $M_{x, y}$ that do not satisfy the properties of $w$-distance in the proof. However, if we replace $M_{x, y}$ with $q(x, y)$ in condition (2) then we obtain the following theorem.

Theorem 2.10. Let $(\mathrm{X}, \mathrm{d}, \preceq)$ be a complete partially ordered quasi metric space equipped with $w$-distance $\mathrm{q}$. Let $\mathrm{f}$ be a self-mapping on $\mathrm{X}$ which has the following conditions:

(1) there exists $x_{0} \in X$ such that $x_{0} \asymp f x_{0}$;

(2) there exist a generalized altering distance function $\psi$, an upper semi-continuous function $\varphi:[0, \infty) \rightarrow[0, \infty)$ and a lower semi-continuous function $\phi:[0, \infty) \rightarrow[0, \infty)$ such that

$$
\psi(q(f x, f y)) \leqslant \varphi(q(x, y))-\phi(q(x, y)) \text { for all } x \asymp y,
$$

where $\varphi(0)=\phi(0)=0$ and $\psi(t)-\varphi(t)+\phi(t)>0$ for all $t>0$;

(3) $f$ is nondecreasing;

(4) (a) either $f$ is continuous or

(b) if $x_{n} \rightarrow x^{*}$, then $x_{n} \asymp x^{*}$. 
Then $\mathrm{f}$ has a fixed point. Moreover, if for each $\mathrm{x}, \mathrm{y} \in \mathrm{X}$ there exists $z \in \mathrm{X}$ which is comparable to $x$ and $y$ then the fixed point is unique.

Proof. Following the proof of Theorem 2.5 for the case, $M_{x, y}=q(x, y)$, we only need to check that if condition (4)-(b) holds, then $f x^{*}=x^{*}$. From (4)-(b) and by using condition (2), we gain

$$
\psi\left(q\left(x_{n+1}, f x^{*}\right)\right)=\psi\left(q\left(f x_{n}, f x^{*}\right)\right) \leqslant \varphi\left(q\left(x_{n}, x^{*}\right)\right)-\phi\left(q\left(x_{n}, x^{*}\right)\right) .
$$

Taking limit as $n \rightarrow \infty$, then

$$
\begin{aligned}
\lim _{n \rightarrow \infty} \psi\left(q\left(x_{n+1}, f x^{*}\right)\right) & \leqslant \lim _{n \rightarrow \infty} \varphi\left(q\left(x_{n}, x^{*}\right)\right)-\lim _{n \rightarrow \infty} \phi\left(q\left(x_{n}, x^{*}\right)\right), \\
\psi\left(\lim _{n \rightarrow \infty} q\left(x_{n+1}, f x^{*}\right)\right) & \leqslant \varphi\left(\lim _{n \rightarrow \infty} \sup q\left(x_{n}, x^{*}\right)\right)-\phi\left(\lim _{n \rightarrow \infty} \inf q\left(x_{n}, x^{*}\right)\right) \\
& =\varphi\left(\lim _{n \rightarrow \infty} q\left(x_{n}, x^{*}\right)\right)-\phi\left(\lim _{n \rightarrow \infty} q\left(x_{n}, x^{*}\right)\right) \\
& =\varphi(0)-\phi(0)=0 .
\end{aligned}
$$

Since $\psi$ is a generalized altering distance function, then $\lim _{n \rightarrow \infty} q\left(x_{n+1}, f x^{*}\right)=0$. So $\lim _{n \rightarrow \infty} q\left(x_{n}, f x^{*}\right)=$ 0 . From Lemma 2.4, if $q\left(x_{n}, x^{*}\right) \rightarrow 0$ and $q\left(x_{n}, f x^{*}\right) \rightarrow 0$, then $d^{s}\left(x^{*}, f x^{*}\right) \rightarrow 0$. Hence $x^{*}=f x^{*}$.

Now, we prove the uniqueness of the fixed point. Assume that $f$ has another fixed point $y^{*}$. From the assumption in theorem, there exists $z \in X$ such that $x^{*} \asymp z$ and $y^{*} \asymp z$. If $z=x^{*}$ or $z=y^{*}$, it is trivial. We assume $z \neq x^{*}$ and $z \neq y^{*}$. Put $z_{0}=z$ and choose $z_{1} \in X$ such that $z_{1}=f z_{0}$. Then we have $z_{0} \asymp x^{*}$. We gain that $f z_{0} \asymp f x^{*}$ or $z_{1} \asymp x^{*}$ by using condition (3) in the theorem. Continuing this process inductively, we get $z_{n} \asymp x^{*}$, for definiteness we suppose $z_{n} \neq x^{*}$ for all $n \in \mathbb{N}$. Similarly, we obtain $z_{n} \asymp y^{*}$ and $z_{\mathrm{n}} \neq \mathrm{y}^{*}$ for all $\mathrm{n} \in \mathbb{N}$.

Next, we show that $\left\{q\left(z_{n}, x^{*}\right)\right\}$ is a decreasing sequence. Suppose to the contrary that $\left\{q\left(z_{n}, x^{*}\right)\right\}$ is a nondecreasing sequence. From condition (2), we have

$$
\psi\left(q\left(z_{n}, x^{*}\right)\right)=\psi\left(q\left(f z_{n-1}, f x^{*}\right)\right) \leqslant \varphi\left(q\left(z_{n-1}, x^{*}\right)\right)-\phi\left(q\left(z_{n-1}, x^{*}\right)\right) \leqslant \psi\left(q\left(z_{n-1}, x^{*}\right)\right) .
$$

Since $\psi$ is a nondecreasing, so $\left(q\left(z_{\mathfrak{n}}, x^{*}\right)\right) \leqslant\left(q\left(z_{\mathfrak{n}-1}, x^{*}\right)\right)$ is a contradiction. Thus, $\left\{q\left(z_{\mathfrak{n}}, x^{*}\right)\right\}$ is a decreasing sequence. That is, there exists $l \geqslant 0$ such that $q\left(z_{n}, x^{*}\right) \rightarrow l$. Now, assume that $l \neq 0$. By using the properties of $\psi, \varphi, \phi$, and taking $n \rightarrow \infty$, we gain $\psi(l) \leqslant \varphi(l)-\phi(l)$. Therefore $\psi(l)-\varphi(l)+\phi(l) \leqslant 0$ is a contradiction with condition (2). Then $l=0$ and $\mathrm{q}\left(z_{\mathrm{n}}, x^{*}\right) \rightarrow 0$.

Similarly, we can deduce that $\mathrm{q}\left(z_{n}, \mathrm{y}^{*}\right) \rightarrow 0$. From Lemma 2.4 , if $\mathrm{q}\left(z_{n}, x^{*}\right) \rightarrow 0$ and $\mathrm{q}\left(z_{n}, \mathrm{y}^{*}\right) \rightarrow 0$, then $d^{s}\left(x^{*}, y^{*}\right) \rightarrow 0$. Thus $x^{*}=y^{*}$. Hence, the fixed point of $f$ is unique.

Remark 2.11. If we set $\mathrm{q}=\mathrm{d}$ for $(\mathrm{X}, \mathrm{d})$ is complete metric space, we gain three results for Theorem 2.10. The results are Shaddad et al. [22] and Rhoades et al. [19] by setting $\psi$ as identity mapping, and Harjani and Sadarangani [10] by setting $\varphi=\psi$ and $\phi$ as a generalized altering distance function. The other important result is if we consider $\varphi=\psi=\mathrm{I}$ (the identity mapping) and $\phi(t)=(1-\alpha) t$, for all $t \in[0, \infty)$, and $\alpha \in[0,1)$, then this theorem is the classical Banach fixed point theorem.

Inspired by the results of $\mathrm{Su}$ [28], we obtain the following corollary with relieve $\phi$ and set $\mathrm{q}=\mathrm{d}$.

Corollary 2.12. Let $(\mathrm{X}, \mathrm{d}, \preceq)$ be a complete partially ordered quasi metric space. Let $\mathrm{f}$ be a self-mapping on $\mathrm{X}$ which has the following conditions:

(1) there exists $x_{0} \in X$ such that $x_{0} \asymp f \chi_{0}$;

(2) there exist a generalized altering distance function $\psi$, and an upper semi-continuous function $\varphi:[0, \infty) \rightarrow$ $[0, \infty)$ such that

$$
\psi(d(f x, f y)) \leqslant \varphi(d(x, y)) \text { for all } x \asymp y,
$$

where $\phi(0)=0$ and $\psi(t)>\varphi(t)$ for all $t>0$; 
(3) $f$ is nondecreasing;

(4) (a) either $f$ is continuous or

(b) if $x_{n} \rightarrow x^{*}$, then $x_{n} \asymp x^{*}$.

Then $\mathrm{f}$ has a fixed point. Moreover, if for each $\mathrm{x}, \mathrm{y} \in \mathrm{X}$ there exists $z \in \mathrm{X}$ which is comparable to $x$ and $y$ then the fixed point is unique.

Remark 2.13. The result of Gordji et al. [7] is a special case of Corollary 2.12 by setting $\psi=\mathrm{I}$ (the identity mapping) and special function $\varphi=\beta(\mathrm{d}(x, y))$ whenever $\beta:[0, \infty) \rightarrow[0,1)$ in complete metric space.

Now, we present an example to illustrate the obtained result given by Theorem 2.5.

Example 2.14. Let $X=\{0\} \cup\left\{\frac{1}{3^{n}}: n \geqslant 1\right\}$, where $(X, d, \leqslant)$ is complete partially ordered quasi metric space with a metric $d$ and usual order $\leqslant$. Consider $w$-distance $q: X \times X \rightarrow[0, \infty)$ defined by $q(x, y)=y$. Let a generalized altering distance function $\psi(t)=\frac{1}{3} t$, an upper semi-continuous function $\varphi(t)=\frac{1}{9} t$ and a lower semi-continuous function $\phi(t)=\frac{1}{27} \mathrm{t}$. Clearly, $\varphi(0)=\phi(0)=0$ and $\psi(t)-\varphi(t)+\phi(t)>0$ for all $\mathrm{t}>0$.

Now, assume that $f: X \rightarrow X$ by $f x=\frac{x}{81}$, for all $x \in X$. If there is $x_{0}=0 \in X$, then $x_{0}=0=\frac{0}{81}=f(0)=$ $f x_{0}$. It is easy to show that $f$ is nondecreasing and continuous. Furthermore, we show that $f$ satisfies condition (2). If $y=0$, then condition (2) holds. Suppose that $y=\frac{1}{3^{m}}$ for any $m$, then we have

$$
\begin{aligned}
M_{x, y} & =\max \left\{q\left(x, \frac{1}{3^{m}}\right), q(x, f x), q\left(\frac{1}{3^{m}}, f\left(\frac{1}{3^{m}}\right)\right), \frac{1}{2}\left[q\left(x, f\left(\frac{1}{3^{m}}\right)\right)-q\left(\frac{1}{3^{m}}, f x\right)\right]\right\} \\
& =\max \left\{\frac{1}{3^{m}}, f x, f\left(\frac{1}{3^{m}}\right), \frac{1}{2}\left[f\left(\frac{1}{3^{m}}\right)-f x\right]\right\} \\
& =\max \left\{\frac{1}{3^{m}}, \frac{x}{81}, \frac{1}{81\left(3^{m}\right)}, \frac{1}{2}\left[\frac{1}{81\left(3^{m}\right)}-\frac{x}{81}\right]\right\} .
\end{aligned}
$$

Now, we have two cases.

(1). Suppose $x=0$, then

$$
M_{x, y}=\max \left\{\frac{1}{3^{m}}, \frac{0}{81}, \frac{1}{81\left(3^{m}\right)}, \frac{1}{2}\left[\frac{1}{81\left(3^{m}\right)}-\frac{0}{81}\right]\right\}=\max \left\{\frac{1}{3^{m}}, \frac{1}{81\left(3^{m}\right)}, \frac{1}{2}\left[\frac{1}{81\left(3^{m}\right)}\right]\right\}=\frac{1}{3^{m}} .
$$

Hence

$$
\begin{aligned}
\psi(q(f x, f y)) & =\psi\left(q\left(\frac{x}{81}, \frac{y}{81}\right)\right)=\psi\left(\frac{y}{81}\right)=\frac{y}{81.3}=\frac{y}{3^{5}}=\frac{1}{3^{5}\left(3^{m}\right)}=\frac{1}{3^{5+m}} \\
& \leqslant \frac{2}{3^{3+m}}=\frac{1}{3^{2+m}}-\frac{1}{3^{3+m}}=\frac{1}{3^{2}\left(3^{m}\right)}-\frac{1}{3^{3}\left(3^{m}\right)} \\
& =\frac{1}{9} \cdot \frac{1}{3^{m}}-\frac{1}{27} \cdot \frac{1}{3^{m}} \\
& =\varphi\left(\frac{1}{3^{m}}\right)-\phi\left(\frac{1}{3^{m}}\right) \\
& =\varphi\left(M_{x, y}\right)-\phi\left(M_{x, y}\right) .
\end{aligned}
$$

(2). Suppose $x=\frac{1}{3^{s}}$. We obtain two cases.

- If $s \geqslant m$, then

$$
\begin{aligned}
M_{x, y} & =\max \left\{\frac{1}{3^{m}}, \frac{x}{81}, \frac{1}{81\left(3^{m}\right)}, \frac{1}{2}\left[\frac{1}{81\left(3^{m}\right)}-\frac{x}{81}\right]\right\} \\
& =\max \left\{\frac{1}{3^{m}}, \frac{1}{81\left(3^{s}\right)}, \frac{1}{81\left(3^{m}\right)}, \frac{1}{2}\left[\frac{1}{81\left(3^{m}\right)}-\frac{1}{81\left(3^{s}\right)}\right]\right\} \\
& =\frac{1}{3^{m}} .
\end{aligned}
$$

Hence we also get the same results with case 1. 
- If $s<m$, then

$$
\begin{aligned}
M_{x, y} & =\max \left\{\frac{1}{3^{m}}, \frac{x}{81}, \frac{1}{81\left(3^{m}\right)}, \frac{1}{2}\left[\frac{1}{81\left(3^{m}\right)}-\frac{x}{81}\right]\right\} \\
& =\max \left\{\frac{1}{3^{m}}, \frac{1}{81\left(3^{s}\right)}, \frac{1}{81\left(3^{m}\right)}, \frac{1}{2}\left[\frac{1}{81\left(3^{m}\right)}-\frac{1}{81\left(3^{s}\right)}\right]\right\} \\
& =\frac{1}{81\left(3^{s}\right)}=\frac{1}{3^{4+s}} .
\end{aligned}
$$

Hence

$$
\begin{aligned}
\psi(\mathrm{q}(\mathrm{f} x, \mathrm{fy})) & =\psi\left(\mathrm{q}\left(\frac{x}{81}, \frac{y}{81}\right)\right)=\psi\left(\frac{y}{81}\right)=\frac{y}{81 \cdot 3}=\frac{y}{3^{5}}=\frac{1}{3^{5}\left(3^{\mathrm{m}}\right)}=\frac{1}{3^{5+m}} \\
& \leqslant \frac{2}{3^{7+s}}=\frac{2}{3^{3+4+s}}=\frac{1}{3^{2} \cdot 3^{4+s}}-\frac{1}{3^{3} \cdot 3^{4+s}} \\
& =\frac{1}{9} \cdot \frac{1}{3^{4+s}}-\frac{1}{27} \cdot \frac{1}{3^{4+s}} \\
& =\varphi\left(\frac{1}{3^{4+s}}\right)-\phi\left(\frac{1}{3^{4+s}}\right) \\
& =\varphi\left(M_{x, y}\right)-\phi\left(M_{x, y}\right) .
\end{aligned}
$$

So, condition (2) in our theorem holds. Thus, all the conditions are satisfied. Moreover we obtain $\lim _{n \rightarrow \infty} f\left(x_{n}\right)=\lim _{n \rightarrow \infty} \frac{x}{81^{n}}=0$.

\section{Common fixed point theorems}

In this section, we prove the common fixed point theorem. An element $x \in X$ is called a common fixed point of mappings $f: X \rightarrow X$ and $g: X \rightarrow X$ if $x=g x=f x$. In 1976, Jungck [13] proved the interesting generalization of contraction mapping principle as follows.

Theorem 3.1. Let $\mathrm{X}$ be a complete metric space. Let $\mathrm{g}$ be a continuous self-map on $\mathrm{X}$ and $\mathrm{f}$ be any self-map on $\mathrm{X}$ that commutes with $\mathrm{g}$. Further, let $\mathrm{f}$ and $\mathrm{g}$ satisfy $\mathrm{f}(\mathrm{X}) \subseteq \mathrm{g}(X)$ and there exists a constant $\lambda \in(0,1)$ such that for every $x, y \in X$

$$
d(f x, f y) \leqslant \lambda d(g x, g y) .
$$

Then $f$ and $g$ have a unique common fixed point.

Recently, Haghi et al. [9] introduced a very useful lemma which we need in our work.

Lemma 3.2 ([9]). Let $\mathrm{X}$ be a nonempty set and $\mathrm{f}: \mathrm{X} \rightarrow \mathrm{X}$ be a function. Then there exists a subset $\mathrm{E} \subseteq \mathrm{X}$ such that $f(E)=f(X)$ and $f: E \rightarrow X$ is one-to-one.

Ciric et al. [5] introduced the following definition which will be needed in the proof of our result.

Definition 3.3 ([5]). Suppose $(X, \preceq)$ is a partially ordered set and $f, g: X \rightarrow X$. $f$ is g-nondecreasing if for all $x, y \in X, g x \preceq g y$ implies $f x \preceq f y$.

Then, from the previous results to fixed point, we will extend for a common fixed point as the way of Shatanawi and Postolache [27]. Now, we are ready to mention our theorem.

Theorem 3.4. Let $(\mathrm{X}, \mathrm{d}, \preceq)$ be a partially ordered quasi metric space equipped with $w$-distance $\mathrm{q}$. Suppose $\mathrm{g}$ be a continuous self-map on $\mathrm{X}$ and $\mathrm{f}$ be any self-map on $\mathrm{X}$ that commutes with $\mathrm{g}$. Further, let $\mathrm{f}$ and $\mathrm{g}$ satisfy $\mathrm{f}(\mathrm{X}) \subseteq \mathrm{g}(\mathrm{X})$ and $\mathrm{g}(\mathrm{X})$ is complete subspace of $\mathrm{X}$ which has the following conditions:

(1) there exists $\mathrm{gx}_{0} \in \mathrm{g}(\mathrm{X})$ such that $\mathrm{gx}_{0} \asymp \mathrm{fx}_{0}$; 
(2) there exist a generalized altering distance function $\psi$, an upper semi-continuous function $\varphi:[0, \infty) \rightarrow[0, \infty)$, and a lower semi-continuous function $\phi:[0, \infty) \rightarrow[0, \infty)$ such that

$$
\psi(q(f x, f y)) \leqslant \varphi\left(m_{x, y}\right)-\phi\left(m_{x, y}\right) \text { for all } x \asymp y,
$$

where $m_{x, y}=\max \left\{q(g x, g y), q(g x, f x), q(g y, f y), \frac{1}{2}[q(g x, f y)-q(g y, f x)]\right\}, \varphi(0)=\phi(0)=0$, and $\psi(t)-$ $\varphi(\mathrm{t})+\phi(\mathrm{t})>0$ for all $\mathrm{t}>0$;

(3) $f$ is g-nondecreasing and continuous.

Then $f$ and $g$ have a common fixed point.

Proof. By Lemma 3.2, there exists $E \subseteq X$ such that $g(E)=g(X)$ and $g: E \rightarrow X$ is one-to-one. We define a continuous mapping $G: g(E) \rightarrow g(X)$ by $G(g x)=f x$. Since $g$ is one-to-one on $E, G$ is well-defined. Now, we show that the function $G$ satisfies all conditions in Theorem 3.4. From the above definition, $G$ is continuous function. Other conditions will be indicated as follows.

(i) We have $g x_{0} \in g(E)$, then $G\left(g x_{0}\right)=f x_{0}$. From condition (1) $g x_{0} \asymp f x_{0}$, we obtain $g x_{0} \asymp G\left(g x_{0}\right)$.

(ii) From the definition of $G$, for all $g x, g y \in g(E)$ we obtain

$$
\psi(q(G(g x), G(g y))) \leqslant \varphi\left(m_{x, y}\right)-\phi\left(m_{x, y}\right) \text { for all } g x \asymp g y,
$$

where $m_{x, y}=\max \left\{q(g x, g y), q(g x, G(g x)), q(g y, G(g y)), \frac{1}{2}[q(g x, G(g y))-q(g y, G(g x))]\right\}, \varphi(0)=$ $\phi(0)=0$, and $\psi(t)-\varphi(t)+\phi(t)>0$ for all $t>0$.

(iii) From Definition 3.3, if $g x \preceq$ gy implies $f x \preceq$ fy for all $x, y \in X$, so that $g x \preceq g y$ implies $G(g x) \preceq$ $G(g y)$ for $g x, g y \in g(E)$, then $G$ is nondecreasing.

From (i)-(iii) above, we conclude that the function $G$ satisfies all conditions in Theorem 2.5. Since $g(E)=$ $g(X)$ is complete and using Theorem 2.5, there exists $x^{*} \in X$ such that $g x^{*}=G\left(g x^{*}\right)=f x^{*}$. Hence $f$ and $\mathrm{g}$ have a common fixed point.

From Remark 2.6 and setting $q=d, \varphi=\psi$, we obtain the following corollaries.

Corollary 3.5. Let $(\mathrm{X}, \mathrm{d}, \preceq)$ be a partially ordered quasi metric space equipped with $w$-distance $\mathrm{q}$. Suppose $\mathrm{g}$ be a continuous self-map on $\mathrm{X}$ and $\mathrm{f}$ be any self-map on $\mathrm{X}$ that commutes with $\mathrm{g}$. Further, let $\mathrm{f}$ and $\mathrm{g}$ satisfy $\mathrm{f}(\mathrm{X}) \subseteq \mathrm{g}(\mathrm{X})$ and $\mathrm{g}(\mathrm{X})$ is complete subspace of $\mathrm{X}$ which has the following conditions:

(1) there exists $\mathrm{gx}_{0} \in \mathrm{g}(\mathrm{X})$ such that $\mathrm{gx}_{0} \asymp \mathrm{fx}_{0}$;

(2) there exist a generalized altering distance function $\psi$, an upper semi-continuous function $\varphi:[0, \infty) \rightarrow[0, \infty)$, and a lower semi-continuous function $\phi:[0, \infty) \rightarrow[0, \infty)$ such that

$$
\psi(q(f x, f y)) \leqslant \varphi\left(\bar{m}_{x, y}\right)-\phi\left(\bar{m}_{x, y}\right) \text { for all } x \asymp y,
$$

where $\bar{m}_{x, y}=\max \{q(g x, g y), q(g x, f x), q(g y, f y)\}, \varphi(0)=\phi(0)=0$, and $\psi(t)-\varphi(t)+\phi(t)>0$ for all $\mathrm{t}>0$;

(3) $f$ is g-nondecreasing and continuous.

Then $\mathrm{f}$ and $\mathrm{g}$ have a common fixed point.

Corollary 3.6. Let $(\mathrm{X}, \mathrm{d}, \preceq)$ be a partially ordered quasi metric space. Suppose $\mathrm{g}$ be a continuous self-map on $\mathrm{X}$ and $f$ be any self-map on $X$ that commutes with $g$. Further, let $f$ and $g$ satisfy $f(X) \subseteq g(X)$ and $g(X)$ is complete subspace of $\mathrm{X}$ which has the following conditions:

(1) there exists $\mathrm{gx}_{0} \in \mathrm{g}(\mathrm{X})$ such that $\mathrm{gx}_{0} \asymp \mathrm{fx}_{0}$; 
(2) there exist a generalized altering distance function $\psi$ and a lower semi-continuous function $\phi:[0, \infty) \rightarrow[0, \infty)$ such that

$$
\psi(d(f x, f y)) \leqslant \psi\left(m_{x, y}\right)-\phi\left(m_{x, y}\right) \text { for all } x \asymp y,
$$

where $m_{x, y}=\max \left\{d(g x, g y), d(g x, f x), d(g y, f y), \frac{1}{2}[d(g x, f y)-d(g y, f x)]\right\}, \phi(0)=0$, and $\phi(t)>0$ for all $\mathrm{t}>0$;

(3) $f$ is g-nondecreasing and continuous.

Then $f$ and $g$ have a common fixed point.

Remark 3.7. The result of Abbas and khan [1] is a special case of our Corollary 3.6 by setting $m_{x, y}=$ $\mathrm{d}(\mathrm{gx}, \mathrm{gy})$ in metric space.

\section{Acknowledgment}

The authors would like to acknowledge the grant UKM Grant DIP-2014-034, and Ministry of Education, Malaysia grant FRGS/ 1/2014/ST06/UKM/01/1. The authors also would like to thank to financial support received from Directorate General of Higher Education (DIKTI), Indonesia.

\section{References}

[1] M. Abbas, M. A. Khan, Common fixed point theorem of two mappings satisfying a generalized weak contractive condition, Int. J. Math. Math. Sci., 2009 (2009), 9 pages. 1, 3.7

[2] R. P. Agarwal, M. A. El-Gebeily, D. O'Regan, Generalized contractions in partially ordered metric spaces, Appl. Anal., 87 (2008), 109-116. 1

[3] C. Alegre, J. Marín, S. Romaguera, A fixed point theorem for generalized contractions involving w-distances on complete quasi-metric spaces, Fixed Point Theory Appl., 2014 (2014), 8 pages. 1, 1, 2.3, 2.4

[4] L. Ćirić, R. P. Agarwal, B. Samet, Mixed monotone-generalized contractions in partially ordered probabilistic metric spaces, Fixed Point Theory Appl., 2011 (2011), 13 pages. 1, 1

[5] L. Cirić, N. Cakić, M. Rajović, J. S. Ume, Monotone generalized nonlinear contractions in partially ordered metric spaces, Fixed Point Theory Appl., 2008 (2008), 11 pages. 3, 3.3

[6] P. N. Dutta, B. Choudhury, A generalisation of contraction principle in metric spaces, Fixed Point Theory Appl., 2008 (2008), 8 pages. 1,1

[7] M. Eshaghi Gordji, H. Baghani, G. H. Kim, A fixed point theorem for contraction type maps in partially ordered metric spaces and application to ordinary differential equations, Discrete Dyn. Nat. Soc., 2012 (2012), 8 pages. 1, 2.13

[8] L. Gholizadeh, R. Saadati, W. Shatanawi, S. M. Vaezpour, Contractive mapping in generalized, ordered metric spaces with application in integral equations, Math. Probl. Eng., 2011 (2011), 14 pages. 1

[9] R. H. Haghi, S. Rezapour, N. Shahzad, Some fixed point generalizations are not real generalizations, Nonlinear Anal., 74 (2011), 1799-1803. 3, 3.2

[10] J. Harjani, K. Sadarangani, Generalized contractions in partially ordered metric spaces and applications to ordinary differential equations, Nonlinear Anal., 72 (2010), 1188-1197. 1, 1, 1.2, 1, 2.11

[11] D. Ilić, V. Rakočević, Common fixed points for maps on metric space with w-distance, Appl. Math. Comput., 199 (2008), 599-610. 1

[12] M. Imdad, F. Rouzkard, Fixed point theorems in ordered metric spaces via w-distances, Fixed Point Theory Appl., 2012 (2012), 17 pages. $1,2.1,2.2,2$

[13] G. Jungck, Commuting mappings and fixed points, Amer. Math. Monthly, 83 (1976), 261-263. 3

[14] O. Kada, T. Suzuki, W. Takahashi, Nonconvex minimization theorems and fixed point theorems in complete metric spaces, Math. Japon., 44 (1996), 381-391. 1, 1.4, 1

[15] M. S. Khan, M. Swaleh, S. Sessa, Fixed point theorems by altering distances between the points, Bull. Austral. Math. Soc., 30 (1984), 1-9. 1

[16] A. Latif, S. A. Al-Mezel, Fixed point results in quasimetric spaces, Fixed Point Theory Appl., 2011 (2011), 8 pages. 1

[17] S. H. Park, On generalizations of the Ekeland-type variational principles, Nonlinear Anal., 39 (2000), 881-889. 1

[18] B. E. Rhoades, Some theorems on weakly contractive maps, Proceedings of the Third World Congress of Nonlinear Analysts, Part 4, Catania, (2000). Nonlinear Anal., 47 (2001), 2683-2693. 1, 1

[19] B. E. Rhoades, H. K. Pathak, S. N. Mishra, Some weakly contractive mapping theorems in partially ordered spaces and applications, Demonstratio Math., 45 (2012), 621-636. 1, 2.11

[20] F. Rouzkard, M. Imdad, D. Gopal, Some existence and uniqueness theorems on ordered metric spaces via generalized distances, Fixed Point Theory Appl., 2013 (2013), 20 pages. 1 
[21] R. Saadati, S. M. Vaezpoura, P. Vetro, B. E. Rhoades, Fixed point theorems in generalized partially ordered G-metric spaces, Math. Comput. Modelling, 52 (2010), 797-801. 1, 1

[22] F. Shaddad, M. S. M. Noorani, S. M. Alsulami, H. Akhadkulov, Coupled point results in partially ordered metric spaces without compatibility, Fixed Point Theory Appl., 2014 (2014), 18 pages. 1, 1, 1.3, 1, 2.11

[23] W. A. Shatanawi, K. K. Abodaye, A. Bataihah, Fixed point theorem through $\Omega$-Distance of Suzuki type contraction condition, Gazi Univ. J. Sci., 29 (2016), 129-133. 1, 1

[24] W. Shatanawi, A. Bataihah, A. Pitea, Fixed and common fixed point results for cyclic mappings of $\Omega$-distance, J. Nonlinear Sci. Appl., 9 (2016), 727-735. 1

[25] W. Shatanawi, A. Pitea, Fixed and coupled fixed point theorems of $\Omega$-distance for nonlinear contraction, Fixed Point Theory Appl., 2013 (2013), 16 pages. 1

[26] W. Shatanawi, A. Pitea, $\Omega$-distance and coupled fixed point in G-metric spaces, Fixed Point Theory Appl., 2013 (2013), 15 pages. 1

[27] W. Shatanawi, M. Postolache, Coincidence and fixed point results for generalized weak contractions in the sense of Berinde on partial metric spaces, Fixed Point Theory Appl., 2013 (2013), 17 pages. 1, 3

[28] Y.-F. Su, Contraction mapping principle with generalized altering distance function in ordered metric spaces and applications to ordinary differential equations, Fixed Point Theory Appl., 2014 (2014), 15 pages. 1, 1.1, 1, 1, 2

[29] F.-F. Yan, Y.-F. Su, Q.-S. Feng, A new contraction mapping principle in partially ordered metric spaces and applications to ordinary differential equations, Fixed Point Theory Appl., 2012 (2012), 13 pages. 1 